\title{
Identification of Compartment- and Process-Specific Molecules Required for "Synaptic Tagging" during Long-Term Potentiation and Long-Term Depression in Hippocampal CA1
}

\author{
Sreedharan Sajikumar, ${ }^{\star}$ Sheeja Navakkode, ${ }^{\star}$ and Julietta U. Frey \\ Leibniz Institute for Neurobiology, Department of Neurophysiology, 39118 Magdeburg, Germany
}

\begin{abstract}
Protein synthesis-dependent forms of hippocampal long-term potentiation (late LTP) and long-term depression (late LTD) are prominent cellular mechanisms underlying memory formation. Recent data support the hypothesis that neurons store relevant information in dendritic functional compartments during late LTP and late LTD rather than in single synapses. It has been suggested that processes of "synaptic tagging" are restricted to such functional compartments. Here, we show that in addition to apical CA1 dendrites, synaptic tagging also takes place within basal CA1 dendritic compartments after LTP induction. We present data that tagging in the basal dendrites is restricted to these compartments. Plasticity-related proteins, partially nonspecific to the locally induced process, are synthesized in dendritic compartments and then captured by local, process-specific synaptic tags. We support these findings in two ways: (1) late LTP/LTD, locally induced in apical or basal (late LTP) dendrites of hippocampal CA1 neurons, does not spread to the basal or apical compartment, respectively; (2) the specificity of the synaptic plasticity event is achieved by the activation of process- and compartmentspecific synaptic tag molecules. We have identified calcium/calmodulin-dependent protein kinase II as the first LTP-specific and extracellular signal-regulated kinase 1/2 as LTD-specific tag molecules in apical dendritic CA1 compartments, whereas either protein kinase A or protein kinase $\mathrm{M} \zeta$ mediates LTP-specific tags in basal dendrites.
\end{abstract}

Key words: long-term potentiation; early LTP; late LTP; synaptic tagging; synaptic cross-tagging; hippocampus

\section{Introduction}

Long-term potentiation (LTP) and long-term depression (LTD) are considered as cellular models for memory. LTP/LTD maintenance requires the synthesis of plasticity-related proteins (PRPs) (Krug et al., 1984; Frey et al., 1988; Matthies, 1989; Otani et al., 1989; Manahan-Vaughan et al., 2000). How PRPs interact only with activated synapses expressing LTP or LTD is fundamental to synapse specificity of the process and can be explained by the "synaptic tagging" hypothesis (Frey and Morris, 1997). Synaptic tagging has also been shown by other laboratories (Barco et al., 2002; Dudek and Fields, 2002; Fonseca et al., 2004; Young and Nguyen, 2005), and it was described for LTD (Kauderer and Kandel, 2000; Sajikumar and Frey, 2004a) and for invertebrates (Casadio et al., 1999).

Recently, the synaptic tagging model has expanded to include functional interactions between LTP and LTD ("cross-tagging") (Sajikumar and Frey, 2004a). It describes the capability of late

\footnotetext{
Received Nov. 14, 2006; revised April 10, 2007; accepted April 10, 2007.

This work was supported by the Volkswagenstiftung I/77 922 and the Deutsche Forschungsgemeinschaft Sonderforschungsbereich 426/B6 to J.U.F. We are grateful to Diana Koch for her excellent technical assistance.

*S.S. and S.N. contributed equally to this work.

Correspondence should be addressed to Dr. Julietta U. Frey, Department for Neurophysiology, Leibniz Institute for Neurobiology, Brenneckestrasse 6, 39118 Magdeburg, Germany. E-mail: frey@ifn-magdeburg.de. DOI:10.1523/JNEUROSCI.4940-06.2007

Copyright $\odot 2007$ Society for Neuroscience $\quad$ 0270-6474/07/275068-13\$15.00/0
}

LTP/LTD in one synaptic input to transform the opposite, protein synthesis-independent early LTD/LTP in an independent synaptic input into its long-lasting form. Cross-tagging expands the repertoire of functional interactions between different synapses and raises the following questions: synaptic tags must mark an activated synapse specifically for either LTP or LTD; which molecules then make tags LTP or LTD specific? Also, PRPs can be process specific, unspecific, and regulatory ("process-specificity" here means its relation to the plasticity-type induced) (Navakkode et al., 2004; Sajikumar et al., 2005b). Thus, can we search for the specificity of the tag complex by using cross-tagging paradigms? If PRPs are not restricted to a synapse, is their synthesis restricted to local dendritic compartments (Martin et al., 2000; Steward and Schuman, 2001; Steward, 2002a,b; Kelleher et al., 2004b; Sweatt, 2004) or can PRP synthesis affect synapses neuron wide? First, evidence for functional compartmentalization has recently been presented by Alarcon et al. (2006). CA1 neurons receive their inputs in apical and basal dendrites from other hippocampal neurons and extrahippocampal structures, such as the amygdala, ventral tegmental area, and others (Amaral and Kurz, 1985; Alkon et al., 1991; Amaral and Witter, 1998; Pikkarainen et al., 1999; Lisman and Grace, 2005). The question arises how mechanisms of input-specificity, local dendritic protein synthesis, and the interaction of different synaptic and modulatory inputs could interact in CA1 neurons to assure integrated informa- 
tion processing. The information from inputs containing spatial, contextual, or relational information, or information with system-relevant "decision" content, can be integratively processed at the target neuron, for instance by processes of synaptic tagging or cross-tagging.

Here, we show that the induction of late LTP sets a processspecific synaptic tag that is mediated by calcium/calmodulindependent kinase II (CaMKII), whereas induction of late LTD sets its LTD tags mediated by mitogen-activated protein kinases (MAPKs) in apical dendrites of hippocampal CA1. In basal dendrites (stratum oriens), we studied processes of synaptic LTP tagging and found that MAPKs and CaMKII are unimportant for setting LTP tags, but either protein kinase A (PKA) or protein kinase $\mathrm{M} \zeta(\mathrm{PKM} \zeta)$ mediate the setting of tags. Furthermore, we found that LTP or LTD induction is restricted to functional compartments in which PRP synthesis occurs. Under the investigated conditions, the PRPs appeared compartment restricted.

\section{Materials and Methods}

We used 282 transverse hippocampal slices $(400 \mu \mathrm{m})$ prepared from 282 male Wistar rats (7 weeks old) as described previously (Frey and Morris, 1997, 1998b; Sajikumar and Frey, 2003, 2004a; Sajikumar et al., 2005a). The animals were killed rapidly by a single blow to the back of the neck using a metallic rod (cervical dislocation) and then decapitated (the whole procedure taking $\sim 3-5 \mathrm{~min}$ ). Slices were incubated in an interface chamber at $32^{\circ} \mathrm{C}$, and the incubation medium [artificial CSF (ACSF)] contained the following (in $\mathrm{mm}$ ): $124 \mathrm{NaCl}, 4.9 \mathrm{KCl}, 1.2 \mathrm{KH}_{2} \mathrm{PO}_{4}, 2.0$ $\mathrm{MgSO}_{4}, 2.0 \mathrm{CaCl}_{2}, 24.6 \mathrm{NaHCO}_{3}, 10$ D-glucose. The carbogen consumption was $18-32 \mathrm{~L} / \mathrm{h}$ (depending on the chamber used), and the flow rate of ACSF was $0.74 \mathrm{ml} / \mathrm{min}$. In all experiments, at least two monopolar lacquer-coated, stainless-steel electrodes ( $5 \mathrm{M} \Omega$; AM Systems, Carlsborg, WA) were positioned within the stratum radiatum of the CA1 region for stimulating two separate independent synaptic inputs, rS1 and rS2 (see Fig. $1 A$ ). In stratum oriens studies, one or two additional stimulating electrodes were positioned in separate inputs (oS3 and oS4) to the same neuronal population. For recording the field EPSP (measured as its slope function) and the population spike (PS) amplitude, two electrodes (5 $\mathrm{M} \Omega$; AM Systems) were placed in the CA1 apical dendritic and cell body layer, respectively, of a single neuronal population to investigate plasticity in the apical dendritic branches, and signals were amplified by a custom-made amplifier. In cases in which tagging in the stratum oriens was investigated, one recording electrode was positioned in the basal dendritic layer between the stimulating electrodes rS3 and rS4 for recording field EPSPs at the basal region. In a few cases, the EPSP portion of the potential recorded with the PS electrode was used to analyze the field EPSP in the stratum oriens. This type of recording revealed comparable results with recordings obtained directly from the dendritic layer of the stratum oriens. The signals were digitized using a CED 1401 analog-todigital converter and analyzed with custom-made software (PWIN). Slices were preincubated for $\geq 4 \mathrm{~h}$, which is critical for reliable long-time recordings of late LTP and late LTD (for more details, see Sajikumar et al., 2005a; Sajikumar and Frey, 2004a).

After the preincubation period, the test stimulation strength was determined for each input to elicit a PS of $40 \%$ of its maximal amplitude for all control and LTD-inducing inputs and 25\% for LTP-inducing inputs. For the determination of the test stimulus intensity in the stratum oriens, a field EPSP of $40 \%$ of its maximal amplitude was determined. For stimulation, biphasic constant-current pulses were used. Late LTD was induced using a strong low-frequency stimulation (SLFS) protocol of 900 bursts [one burst consisted of three stimuli at $20 \mathrm{~Hz}$, and the interburst interval was $1 \mathrm{~s}$ (i.e., $\mathrm{f}=1 \mathrm{~Hz}$; stimulus duration, $0.2 \mathrm{~ms} /$ half wave; total number of stimuli, 2700)]. This stimulation pattern produced a stable late LTD in vitro for $\geq 8 \mathrm{~h}$. In experiments in which a weaker induction of LTD was investigated, a weak low-frequency stimulation protocol (WLFS) was used consisting of 900 pulses at a frequency of $1 \mathrm{~Hz}$, an impulse duration of $0.2 \mathrm{~ms} /$ half wave, with 900 total stimuli. Late LTP was induced using three stimulus trains of 100 pulses ["strong" tetanus
(STET), $100 \mathrm{~Hz}$; duration, $0.2 \mathrm{~ms} /$ polarity; intertrain intervals, $10 \mathrm{~min}]$. Early LTP was induced using a weak tetanization (WTET) protocol consisting of one $100 \mathrm{~Hz}$ train (21 biphasic constant-current pulses for tetanization (TET) in the stratum radiatum or 14 pulses for stratum oriens; pulse duration per half wave, $0.2 \mathrm{~ms}$; stimulus intensity for TET, $40 \%$ of maximal field EPSP). The PS and the slope of the field EPSP were monitored on-line. For clarity, the field EPSP data are shown because the two recorded parameters demonstrated similar time courses in the experiments, except in cases in which the PS was abolished during LTD. The baseline was recorded for $\geq 60 \mathrm{~min}$ before LTP/LTD induction; four 0.2 $\mathrm{Hz}$ biphasic constant-current pulses $(0.1 \mathrm{~ms}$ per polarity) were used for baseline recording and testing $1,3,5,11,15,21,25$, and 30 min posttetanus or 21, 25, and $30 \mathrm{~min}$ post-LFS and thereafter once every $15 \mathrm{~min}$ up to $8 \mathrm{~h}$ maximum.

The CaMKII inhibitors KN-62 (1-[NO-bis-1,5-isoquinolinesulfonyl]$\mathrm{N}$-methyl-L-tyrosyl-4-phenylpiperazine; Calbiochem, La Jolla, CA) and AIP (autocamtide-2-related inhibitory peptide; Calbiochem) were used at a concentration of $5 \mu \mathrm{M}$, specific for CaMKII, dissolved in DMSO [the final concentration of DMSO was $0.1 \%$, a concentration which had no effect on the basal synaptic transmission (Navakkode et al., 2004)] as a stock solution $(10 \mathrm{mM})$, and was stored at $-20^{\circ} \mathrm{C}$. The required volume containing the final concentration of $5 \mu \mathrm{M}$ was dissolved in ACSF immediately before bath application. Stock solutions of the MEK inhibitors, 1,4-diamino-2,3-dicyano-1,4-bis[2-amino-phenylthio]butadiene (U0126; Promega, Madison, WI) and 2-(2-amino-3-methoxyphenyl)4H-1-benzopyran-4-one (PD 98059; Calbiochem) were prepared in DMSO at a $10 \mathrm{~mm}$ stock solution, and the required volume containing the final concentration of $20 \mu \mathrm{M}$ was dissolved in ACSF immediately before bath application (final concentration of DMSO was 0.1\%). Anisomycin (25 $\mu \mathrm{M}$; Sigma, St. Louis, MO) and emetine $(20 \mu \mathrm{M}$; Tocris, Ellisville, MO) were dissolved in ACSF. The PKA inhibitor KT5720 (Calbiochem) was used at a concentration of $1 \mu \mathrm{M}$ (dissolved in DMSO to a final concentration of $0.1 \%$ ). The myristoylated pseudosubstrate peptide, ZIP (myr-SIYRRGARRWRKL-OH; Biosource, Camarillo, CA) was prepared in distilled water as a stock solution $(10 \mathrm{mM})$ and stored at $-20^{\circ} \mathrm{C}$. The required volume containing the final concentration of $1 \mu \mathrm{M}$ was dissolved in ACSF immediately before bath application.

The average values of the PS amplitude (measured in millivolts) and the slope function of the field EPSP (millivolts per milliseconds) per time point were analyzed using the Wilcoxon signed rank test when compared within one group, or the Mann-Whitney $U$ test when data were compared between groups; $p<0.05$ was considered as statistically significantly different. The nonparametric test was used because the analyses of prolonged recordings do not allow the use of parametric tests. Furthermore, the sample sizes did not always guarantee a Gaussian normal distribution of the data per series.

\section{Results}

\section{Inhibition of CaMKII or MAPKs on LTP and LTD}

There is considerable evidence that CaMKII as well as MAPKs are critical for the induction of persistent LTP as well as LTD (Malinow et al., 1988, 1989; Stevens et al., 1994; Mayford et al., 1995; Stanton and Gage, 1996; English and Sweatt, 1997; Bortolotto and Collingridge, 1998; Schnabel et al., 1999; Winder et al., 1999; Lee et al., 2000; Dudek and Fields, 2001; Giovannini et al., 2001; Komiyama et al., 2002; Miller et al., 2002; Kelleher et al., 2004a; Sweatt, 2004). In a first set of experiments, we have reproduced these findings to show that our preparations functionally resemble that of others. The application of specific CaMKII or MAPK [MEK-dependent extracellullar-signal-regulated kinases (ERK)] inhibitors block the induction of late LTP/LTD if applied during its induction (Fig. $1 B, D, F, H$ ). Early LTP/LTD was phenotypically unaffected in its time course, although we cannot exclude influences of the drugs on intracellular events less detectable with our methods. Thus, U0126 caused a moderate inhibitory effect on the amplitude of early LTD induction (Fig. $1 \mathrm{H}$, filled circles) as follows: depression from baseline by $29.22 \pm 5.6 \% 1 \mathrm{~min}$ after 
A

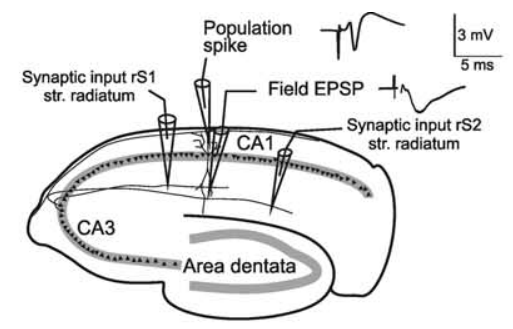

B

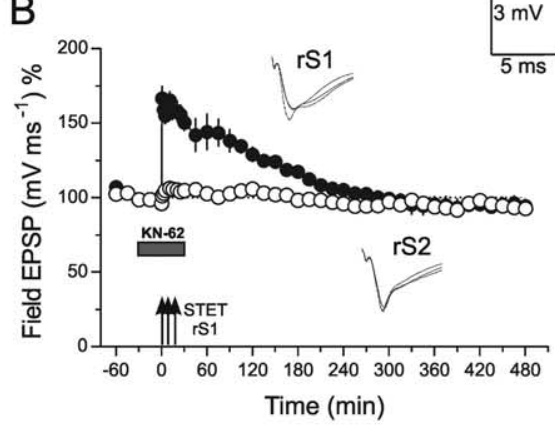

D

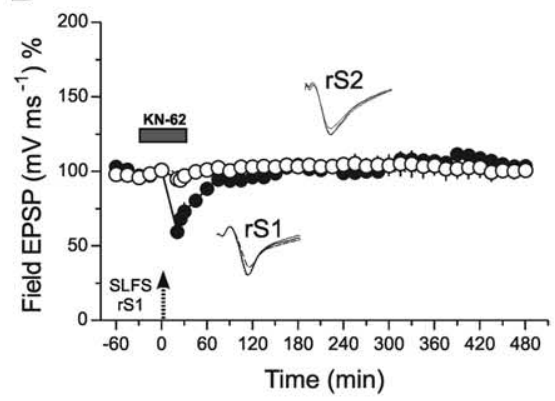

$\mathrm{F}$

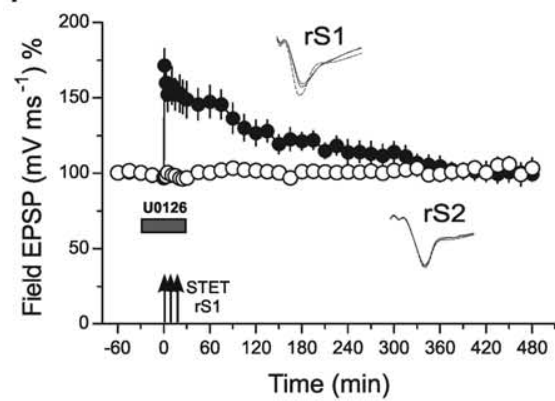

$\mathrm{H}$

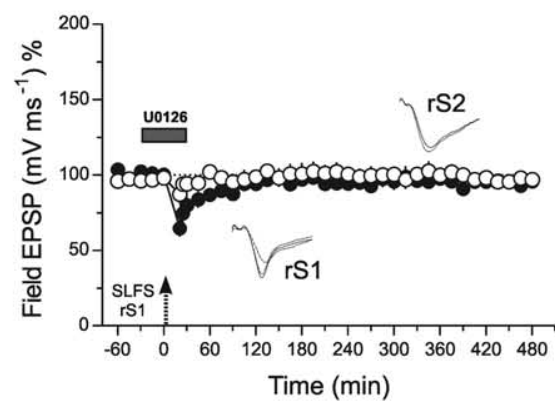

C

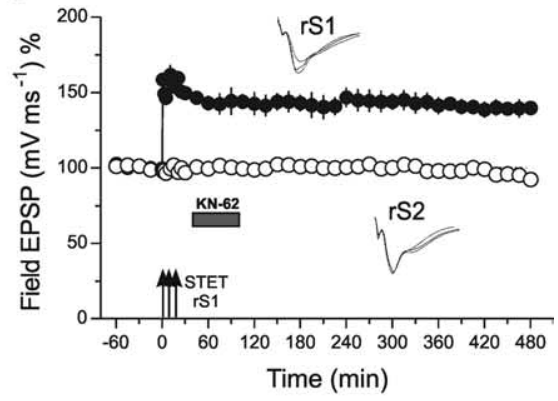

$\mathrm{E}$

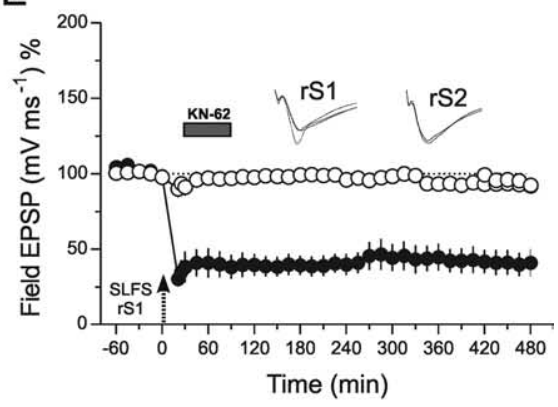

G

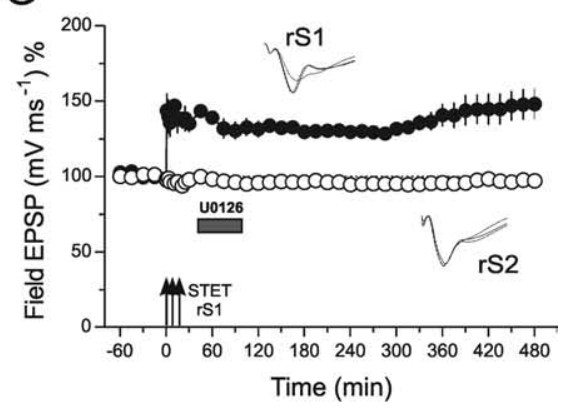

I

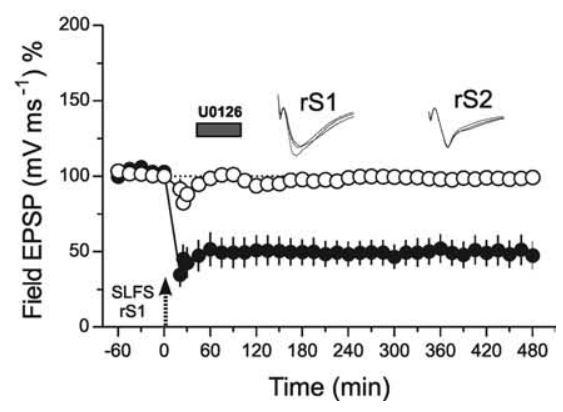

Figure 1. The effect of CaMKII or ERK inhibition on LTP and LTD in hippocampal CA1 neurons. $A$, The schematic location of the electrodes to stimulate two separate synaptic inputs rS1 and rS2 in stratum (str.) radiatum in rat hippocampal slices in vitro. The analog traces near to the recording electrodes show representative examples of recorded potentials. $\boldsymbol{B}$, The action of the CaMKII

LTD induction and in the presence of U0126 when compared with a depression by $56.89 \pm 7.9 \%$ from baseline after LTD induction without U0126 in Fig. 1 I (filled circles). This U0126-related effect on LTD induction could be because of a distinct early involvement of local ERKdependent protein synthesis during the induction and early maintenance of LTD (Navakkode et al., 2005). However, if the drugs were applied after the induction of the late event, they were ineffective in preventing the expression of LTP/LTD (Fig. $1 C, E, G, I)$. Thus, both kinases are required for processes inducing late LTP as well as late LTD. Comparing the effect of the drugs on the level of induction of either LTP or LTD, we found a direct, still unknown influence of the kinase inhibitors on LTD but not LTP; the reason for this remains to be investigated.

\section{Inhibition of CaMKII or MAPKs and synaptic tagging}

In a second set of experiments, we investigated the role of CaMKII and MAPKs on processes of synaptic tagging during LTP or LTD. To do so, we first used the following protocol: we induced late LTP or late LTD by a repeated STET or a complex SLFS (see Materials and Methods) in synaptic input $\mathrm{rS} 1$ within the stratum radiatum (Fig. 2, filled circles). Fifteen minutes later, KN-62, a specific CaMKII inhibitor, was applied for the following $1 \mathrm{~h}$. Fortyfive minutes after the induction of late LTP or late LTD in rS1, late LTP or late LTD in a second independent synaptic input rS2 was triggered (Fig. 2, open circles), but now under the influence of the CaMKII inhibitor. Our initial working hypothesis was that the investigated kinases could initiate the synthesis of PRPs. Thus, we blocked the kinases similar to protein

$\leftarrow$

inhibitor, KN-62, on late LTP in rS1 if applied during its induction (filled circles). The drug was applied $30 \mathrm{~min}$ before TET for a total of $1 \mathrm{~h}$. Open circles represent the time course of the control input rS2. $n=4$. In $C$, the same stimulation protocol was used as in $\boldsymbol{B}$; however, the inhibitor was applied $15 \mathrm{~min}$ after LTP induction in $\mathrm{rS} 1$ and remained in the bath for $1 \mathrm{~h}$ $(n=4)$. $\boldsymbol{D}(n=3)$ and $\boldsymbol{E}(n=4)$ represent experiments similar to those in $\boldsymbol{B}$ and $\boldsymbol{C}$, with the exception that instead of STET, an SLFS was applied to rS1 to investigate a possible effect of the drugs on processes of LTD. Panels $\boldsymbol{F}(n=6), \boldsymbol{G}$ $(n=3), \boldsymbol{H}(n=4)$, and $\boldsymbol{I}(n=5)$ show adequate series of experiments as in $\boldsymbol{B}-\boldsymbol{E}$, but with differences in the blockers used; here, the MEK inhibitor U0126 was applied instead of the CaMKII inhibitor. Gray boxes represent the time of drug application. The inlets in each graph represent typical analog traces per stimulated input $30 \mathrm{~min}$ before (dotted line), 30 min after (broken line), and 60 min after (full line) TET/lowfrequency stimulation. Error bars indicate SEM. 

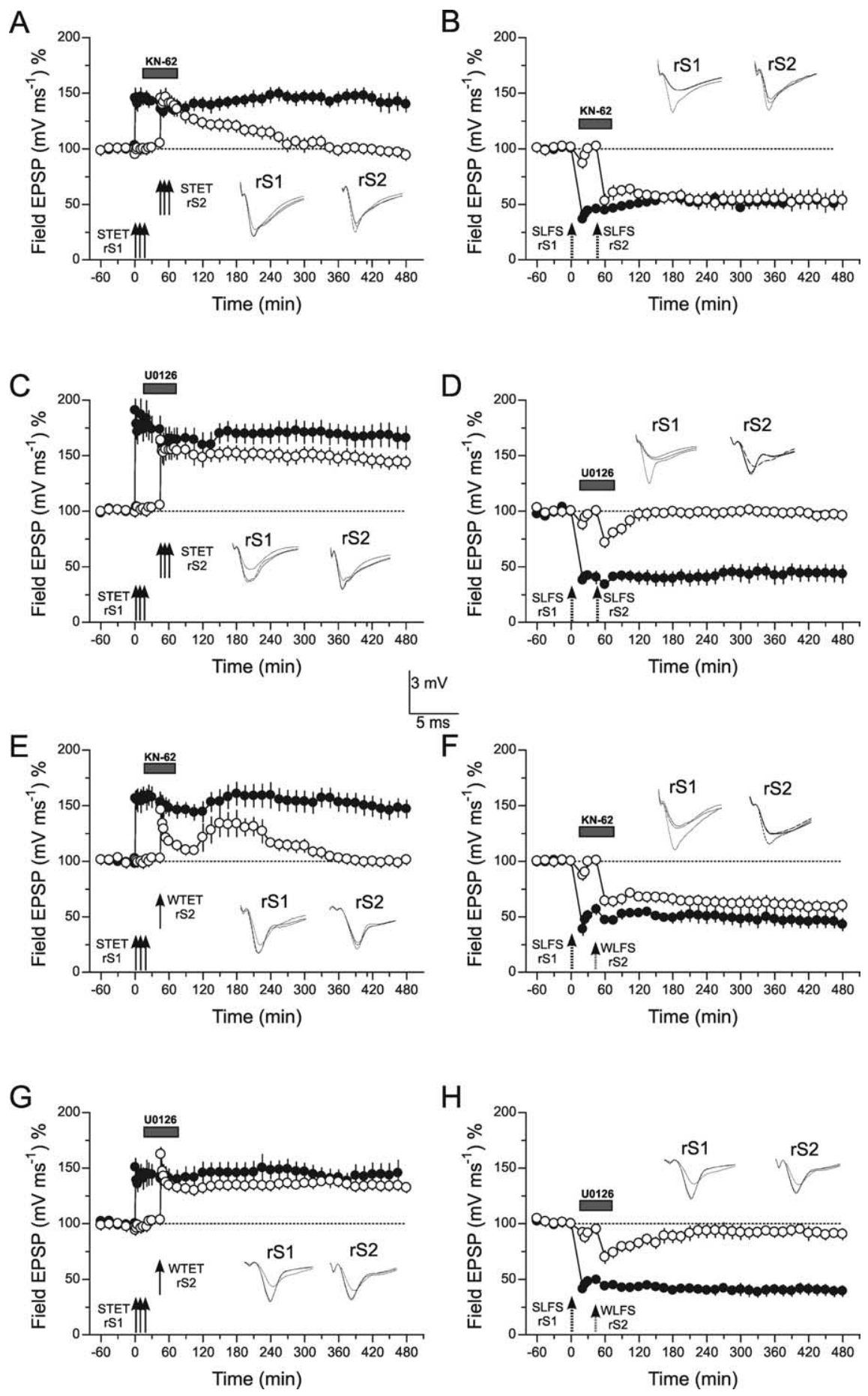

Figure 2. Role of CaMKII and ERKs for synaptic tagging during LTP and LTD. A, The time course of late LTP induced in rS1 (filled circles) by a STET (arrows indicate the time points of STET) followed by the application of the CaMKII inhibitor KN-62 15 min after the first tetanus that remained in the bath for $1 \mathrm{~h}$. Forty-five minutes after the first tetanus in $\mathrm{r}$ S1, an additional STET was applied to $\mathrm{rS} 2$ in the presence of KN-62 (open circles; $n=6$ ). $\boldsymbol{B}$, The same experiment as in $\boldsymbol{A}$; however, instead of late LTP, an SLFS was applied to induce LTD $(n=6)$. C, $\boldsymbol{D}$, Experiments similar to those in $\boldsymbol{A}$ and $\boldsymbol{B}$, except that the MEK inhibitor U0126 was applied (in both series; $n=6$ ). $\boldsymbol{E}, \boldsymbol{F}$, A set of experiments similar to those in $\boldsymbol{A}$ and $\boldsymbol{B}$, except that instead of a strong stimulation to rS2, a WTET in $\boldsymbol{E}$ (WTET, $n=8$ ) or WLFS to S2 in $\boldsymbol{F}(n=7)$ was delivered. $\boldsymbol{G}$ and $\boldsymbol{H}$ represent experiments similar to those in $\boldsymbol{E}$ and $\boldsymbol{F}$ with the difference that instead of the (aMKII inhibitor, an ERK inhibitor was applied (both series, $n=7$ ). Error bars indicate SEM. Symbols and analog traces as in Figure 1.
KN-62, prevented synaptic tagging for $\mathrm{rS} 2$ during LTP (Fig. 2A) but not LTD (Fig. $2 B)$, whereas inhibition of ERKs prevented synaptic tagging in $\mathrm{rS} 2$ for LTD (Fig. 2D) (potentials in rS2 returned to baseline levels within $60 \mathrm{~min}$ after LTD induction) but not for LTP (Fig. 2C).

In addition to the above protocol and to further investigate the specificity of the two kinases for LTP or LTD, we also used a "strong before weak" protocol (Frey and Morris, 1998b; Sajikumar and Frey, 2004a). Here, we induced a strong late event in $\mathrm{rS} 1$ followed by the induction of an early event in $\mathrm{rS} 2$ within a brief time window. Induction of a late event in $\mathrm{rS} 1$ both sets its transient protein synthesisindependent synaptic tag and activates the synthesis of synapse-unspecific PRPs. These PRPs can be captured by synaptic tags at other synaptic inputs in rS2 within the stratum radiatum [for example, at those inputs where early LTP or early LTD was induced (i.e., a protein synthesisindependent transient form of LTP/LTD that sets its own synaptic tag)] and thus, transforming its normally transient plastic form into a long-lasting form of LTP or LTD (Frey and Morris, 1998b; Sajikumar and Frey, 2004a). If the kinase inhibitors were applied during the weak stimulation of input rS2 [single WTET for early LTP or a WLFS for early LTD in rS2], we obtained similar results as presented in Figure $2 A-D$ with a few differences in the LTP series. Although synaptic tagging in $\mathrm{rS} 2$ does not occur (i.e., early LTP in rS2 is not transformed into late LTP), the early LTP in $\mathrm{rS} 2$ shows an intermediate reduction immediately after TET for $1 \mathrm{~h}$ (Fig. 2E, open circles). This could be explained by the involvement of CaMKII-dependent processes during depotentiation-like (Sajikumar and Frey, 2004b) or metaplastic phenomena of LTP (Tompa and Friedrich, 1998), when preceded by a strong event in a second independent input. Alternatively, CaMKII-dependent processes could be involved in a yet unknown mechanism required for expressing a specific intermediate, protein synthesisindependent phase of LTP (Winder et al., 1998). Thus, the strong before weak protocol revealed a similar result as above: CaMKII-dependent processes are involved in LTP but not LTD tagging, whereas ERKdependent processes are required for LTD but not LTP tagging. synthesis in the original tagging experiments (Frey and Morris, 1997; Sajikumar and Frey, 2004a). Interestingly, we obtained different outcomes for the two kinase inhibitors for LTP and LTD. As shown in Figure 2, the application of the CaMKII inhibitor,
Inhibition of CaMKII or ERKs and synaptic cross-tagging In the next series of experiments, we investigated the role of CaMKII- and ERK1/2-dependent activity on processes of synaptic cross-tagging. We have recently shown that within apical den- 
drites of pyramidal CA1 neurons, the induction of a late protein synthesisdependent LTP or LTD can transform its opposite transient form of plasticity into its long-lasting form. Thus, the induction of late LTP (in rS1) can transform early LTD into late LTD (in rS2), or late LTD (in rS1) can prolong early LTP into late LTP (in rS2) if the two events are induced within a time window, as described for synaptic tagging (Sajikumar and Frey, 2004a). Thus, LTD tags or LTP tags in rS2 can capture PRPs whose synthesis was originally initiated by the induction of late LTP or late LTD in rS1. These results allowed us to investigate two predictions: (1) the tags must be process-specific, and (2) the PRPs are synthesized as a pool of proteins that may contain process-specific as well as process-unspecific proteins (Kelleher et al., 2004b; Sajikumar and Frey, 2004a; Sajikumar et al., 2005b). Recently, we identified $\mathrm{PKM} \zeta$ as the first process-specific PRP and the phosphodiesterase type $4 \mathrm{~B} 3$ as a process-unspecific, regulatory PRP (Ahmed and Frey, 2003; Ahmed et al., 2004; Sajikumar et al., 2005b). However, the identity of the molecules that make the local synaptic tag complex specific for either LTP or LTD remained to be investigated.

Figure $3 A, B$ represent experiments in which the protein synthesis-dependent late LTD was induced in rS1 (filled circles) followed by the induction of a protein synthesis-independent early LTP in rS2 (open circles). Under normal circumstances, early LTP in rS2 would have been transformed into late LTP by processes of cross-tagging (i.e., cross-capturing of PRPs originally provided by the late LTD in rS1) (Sajikumar and Frey, 2004a). However, induction of late LTD in rS1 followed by the application of the CaMKII inhibitors (Fig. 3A, KN62; Fig. 3B, AIP) and induction of early LTP in rS2 prevented the transformation of early into late LTP in rS2. Similarly, as in experiments presented in Figure $2 E$, an early transient inhibition of early LTP in rS2 after late LTD in rS1 was observed that supports our hypothesis that CaMKII has multiple actions on the different forms of LTP including metaplastic events (see above).

In contrast, in the opposite experiment (Fig. $3 C, D$ ) (i.e., when the induction of late LTP in rS1 was followed by the application of the CaMKII inhibitors and early LTD in rS2), the transformation process from early into late LTD in $\mathrm{rS} 2$ was not prevented. Thus, PRPs synthesized via late LTP in rS1 could be cross-captured by the
A

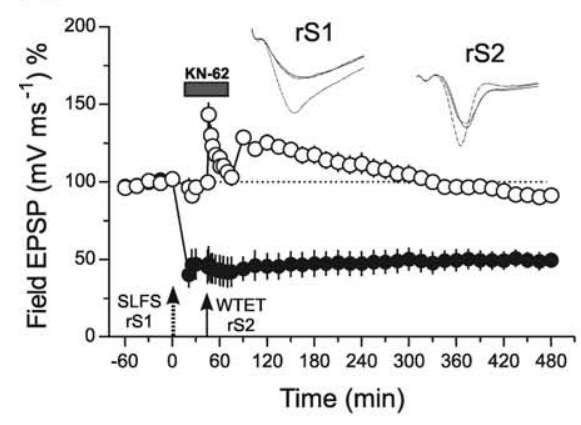

B
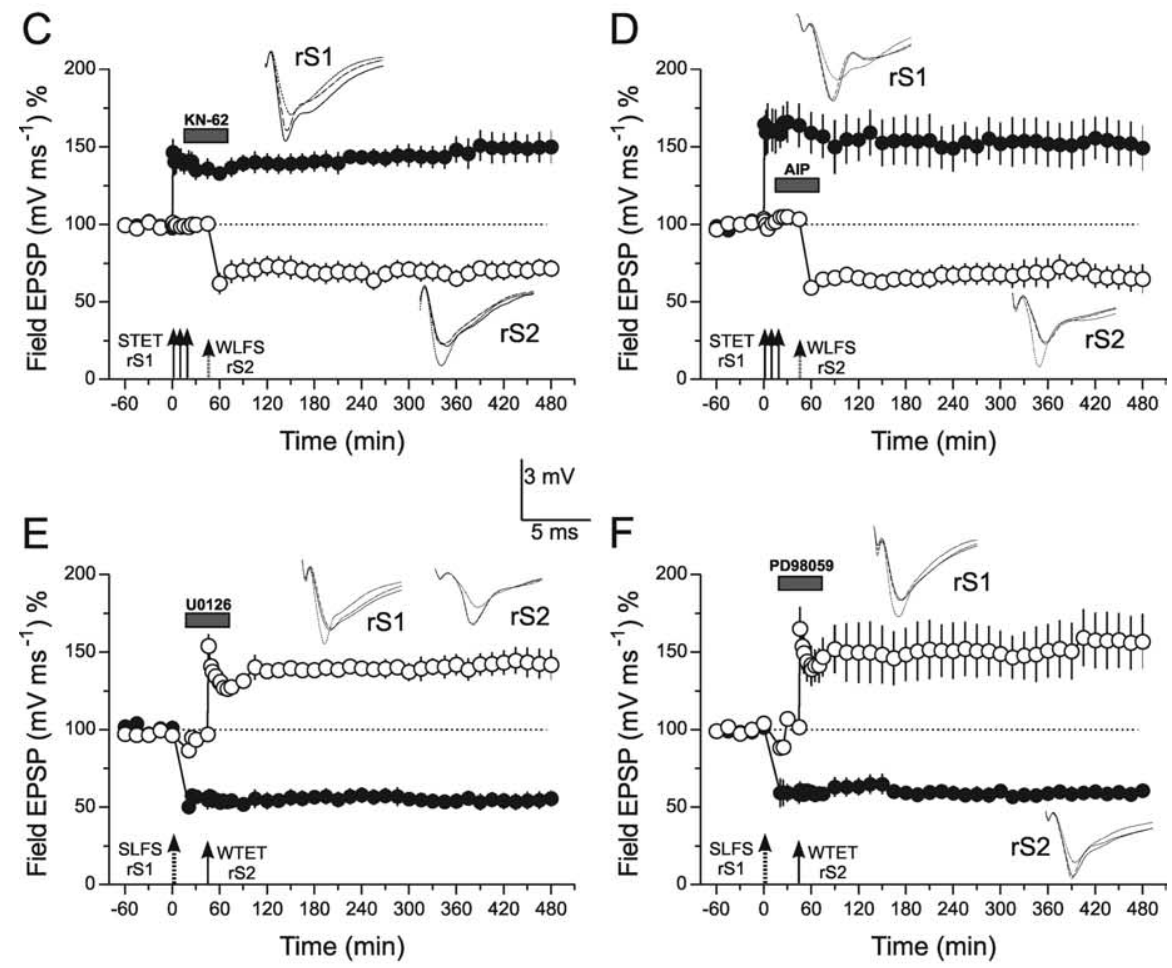

G

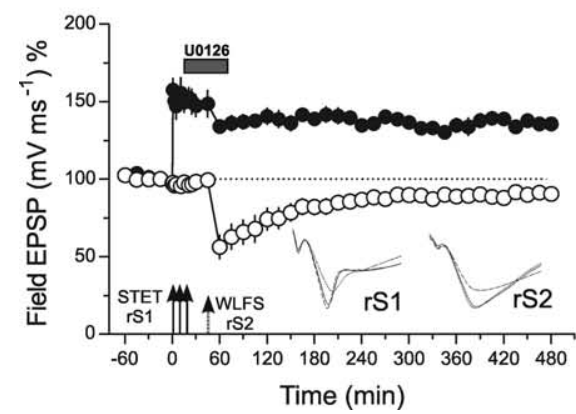

$\mathrm{H}$

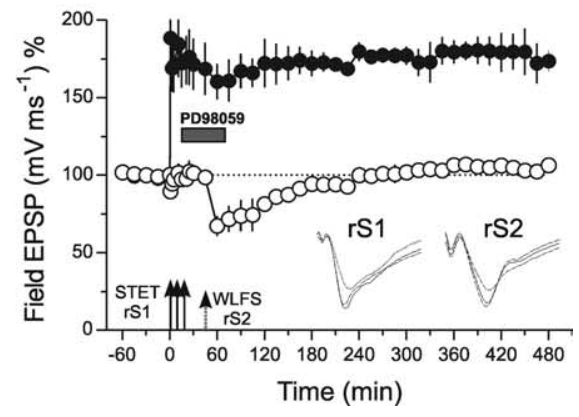

Figure 3. Role of CaMKII and ERKs for synaptic cross-tagging between LTP and LTD. $A, B$, Synaptic cross-tagging experiment (Sajikumar and Frey, 2004a) between late LTD in rS1 (filled circles) and early LTP in rS2 (open circles), and the influence of two structurally different CaMKII inhibitors, KN-62 $(\boldsymbol{A} ; n=6)$ or AIP $(\boldsymbol{B} ; n=6)$ on these processes. SLFS was applied to rS1 (filled circles) followed by the application of the drugs $15 \mathrm{~min}$ later that remained in the bath for the next hour. WTET was applied to $\mathrm{rS2}$ (open circles) 45 min after STET to $\mathrm{rS1}$ and under the influence of the inhibitors. $\ln \boldsymbol{C}(n=6)$ and $\boldsymbol{D}(n=5)$, the opposite order of induction of the plasticity events compared with $\boldsymbol{A}$ and $\boldsymbol{B}$ were investigated. Here, STET was applied first to rS1 (filled circles), followed by the application of the drugs and WLFS to rS2 (open circles). $\boldsymbol{E}(n=7), \boldsymbol{F}(n=4), \boldsymbol{G}(n=7)$, and $\boldsymbol{H}(n=3)$ resemble the experiments from $\boldsymbol{A} \boldsymbol{- D}$, with the exception that instead of the CaMKII inhibitors, two structurally different ERK inhibitors were investigated. Error bars indicate SEM. Symbols and analog traces are as in Figure 1. 
early LTD rS2 input in the absence of CaMKII activity. If CaMKII would be directly involved in the synthesis of PRPs, the crosstagging should not have been observed. Therefore, CaMKII is most likely involved in the tagging process specific for LTP rather than on the local synthesis of PRPs required for the first $8 \mathrm{~h}$ of the long-lasting plastic event. The latter, however, does not necessarily exclude which CaMKII is involved in local protein synthesisdependent processes that might be required for tagging but are phenotypically not detected by the application of protein synthesis inhibitors.

The next experiments presented in Figure $3 E-H$ were performed to investigate the role of ERKs during cross-tagging. Therefore, we used two different MAPK inhibitors (Frey et al., 1993; Frey and Morris, 1998a; Navakkode et al., 2005). The application of these inhibitors were ineffective in interfering with processes of cross-tagging from late LTD in $\mathrm{rS} 1$ (Fig. $3 E, F$, filled circles) to early LTP induced in rS2 (Fig. $3 E, F$, open circles). Thus, PRPs synthesized via late LTD in $\mathrm{rS} 1$ could be captured by LTP tags set in rS2 by cross-tagging processes. In the next experiments (Fig. 3G,H), late LTP was induced in rS1 (filled circles) followed by the application of the ERK inhibitors and early LTD in rS2. Interestingly, the transformation from early into late LTD was prevented under these circumstances, potentials in rS2 returned to baseline within 2-3 h after WLFS, indicating that ERKs are required for activation of LTD but not LTP tags.

\section{Tagging is locally restricted}

We now investigated whether PRPs synthesized locally at the apical or basal dendrites could be captured by synapses in other dendritic compartments (i.e., in the basal or apical dendrites, respectively). To do so, we first determined specific stimulation protocols to induce different forms of LTP in synapses in the basal dendrites (stratum oriens). However, field stimulation in the stratum oriens may also partially activate CA1 axons (i.e., activating antidromically the target cells). This somatic stimulation could lead to a facilitation of plastic events in the stratum radiatum (Dudek and Fields, 2002; Adams and Dudek, 2005). Another possibility is that the requirements for inducing prolonged forms of plastic events in the basal dendrites may differ from those in the apical dendrites (LTP; see below, LTP in basal CA1 dendrites). To avoid these interferences, we first determined specific stimulation protocols that induce different forms of LTP in the stratum oriens synapses. Figure $4 B$ represents the time course of late LTP at synapses of basal dendrites (Fig. $4 A$, oS3). In general, the time course of late LTP in basal dendrites (Fig. $4 B$, filled triangles) is comparable with that obtained in the apical dendrites after repeated TET (for details of the stimulation protocol in the basal dendrites, see Materials and Methods). Late LTP in oS3 is input specific (i.e., it does not influence control potentials in a separate synaptic input rS1 in the apical dendrites). By stimulation with a single tetanus, early LTP in oS3 can also be induced (Fig. 4C). Early LTP in the basal dendrites, however, seems to be more robust compared with early LTP in the apical dendrites. Potentials in oS3 were statistically significantly potentiated up to $225 \mathrm{~min}$ [when compared with control oS4 inputs (Fig. 4C, open triangles); Mann-Whitney $U$ test; $p<0.05$ ] and up to $240 \mathrm{~min}$ when compared with its own baseline before TET ( $p<0.05$; Wilcoxon signed rank test), and before they returned to baseline levels. Late LTP in oS3 can be blocked in its maintenance by the application of protein synthesis inhibitors (anisomycin, emetine) during its induction (i.e., potentials return to baseline within 2-3 h) (Fig. 4D,E) [LTP in oS3 in Fig. $4 D$ (anisomycin) was statistically different from control potentials ob- tained in input oS4 up to $105 \mathrm{~min}$ (open triangles); Mann-Whitney $U$ test; $p<0.05$ ] and up to 90 min when compared with its own baseline ( $p<0.05$; Wilcoxon test). LTP in oS3 under emetine was statistically different up to $135 \mathrm{~min}$ when compared with control input oS4 (Fig. 4E, open triangles) (Mann-Whitney $U$ test; $p<0.05$ ), and the potentiation was detectable up to $105 \mathrm{~min}$ when compared with its own baseline before TET $(p<0.05$; Wilcoxon test).

Taking into account the similar properties of late LTP in oS3, we then investigated whether synaptic tagging (i.e., capturing of PRPs) is restricted to local dendritic compartments or whether PRPs are spread across the neuron. We therefore induced a protein synthesis-dependent late LTP at the basal dendrites (oS3) (Fig. $4 F$, filled triangles), followed by the induction of a normally protein synthesis-independent early LTP in both rS1 and rS2 at apical dendritic compartments (Fig. $4 F$, filled and open circles). Early LTP in the latter inputs were not transformed into late LTP by late LTP in oS3, suggesting that PRPs produced in basal dendrites required to stabilize LTP in oS3 are unable to reach tagged synapses at apical dendrites. To check that tagging is not negatively influenced in the stratum radiatum by the stratum oriens stimulation [either early LTP induction or control stimulation in the oriens (Young and Nguyen, 2005)], we induced late LTP in apical rS1 (Fig. 4G, filled circles), followed by the induction of early LTP in apical rS2 (Fig. 4G, open circles) and in basal oS3 (Fig. 4G, filled triangles). Early LTP in the apical rS2 was transformed into late LTP, whereas early LTP in the basal oS3 was unaffected in its duration, suggesting that stimulation and the induction of LTP in the stratum oriens does not prevent processes of synaptic tagging within the apical dendrites. If early LTP was induced in the basal inputs oS3 (Fig. $4 H$, filled triangles) and oS4 (Fig. $4 H$, open triangles) before induction of late LTP in the stratum radiatum ( $\mathrm{rS} 1$ ) (Fig. $4 \mathrm{H}$, filled circles), early LTP in the stratum oriens could not benefit from late LTP in stratum radiatum, resulting in a decremental LTP in the basal inputs. These data support the previous finding that LTP is restricted to functional dendritic compartments.

In a next set of experiments, we studied whether synaptic cross-tagging is affected by the cross-compartmentalized induction of various plastic events. As shown in Figure 4I, we induced late LTD in apical rS1 (filled circles), followed by early LTP in apical rS2 (open circles) and in basal oS3 (filled triangles). Early LTP in apical rS2 but not in basal oS3 was transformed into late LTP, suggesting that rS1 LTD-induced synthesis of PRPs was locally restricted to the apical dendrites where they could be captured by the compartment-specific rS2 tags but not the basal oS3 tags. Thus, processes of both synaptic tagging and cross-tagging are restricted to functional dendritic compartments under these conditions

The next questions we investigated was whether synaptic tagging can be detected during LTP in the stratum oriens and if so, whether tag molecules are identical or different in the stratum oriens when compared with the stratum radiatum (Figs. 5, 6).

\section{LTP in basal CA1 dendrites}

Most hippocampal LTP studies have been performed in synapses within the stratum radiatum. Because LTP is restricted to dendritic compartments, the question arises if the same intracellular cascades are involved in processes of synaptic tagging and thus maintaining LTP in apical as well as in basal dendritic compartments. Therefore, we have induced late LTP in basal synaptic inputs under the influence of various inhibitors (Figs. $4 B-E, 5$ ), but also of the kinases (Fig. 5), for which we had shown a tag- 
A

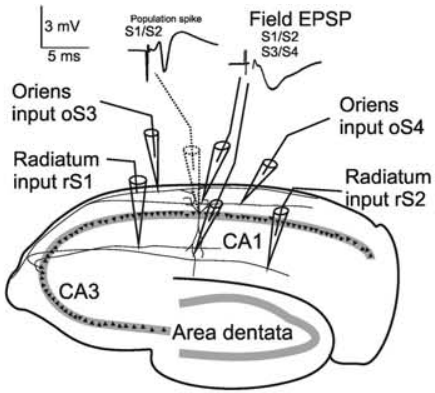

C
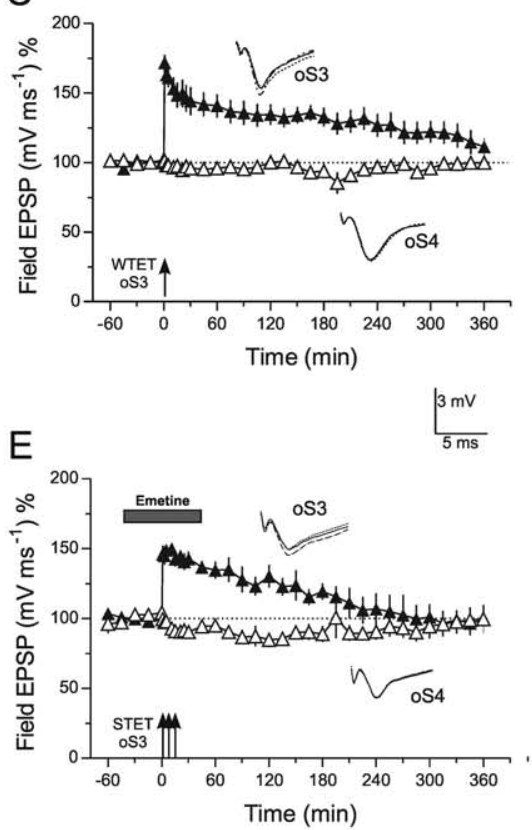

G

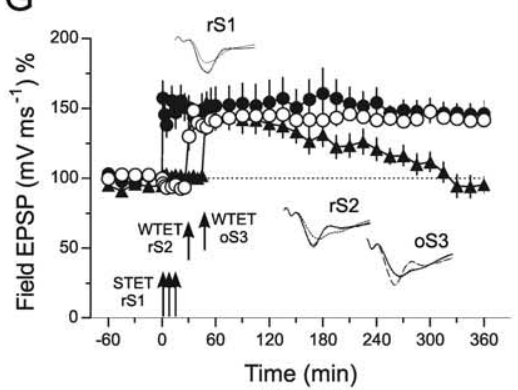

I

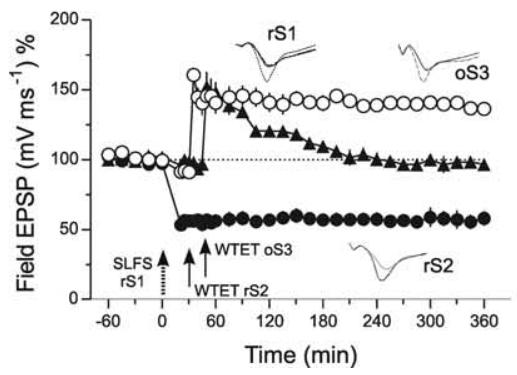

B

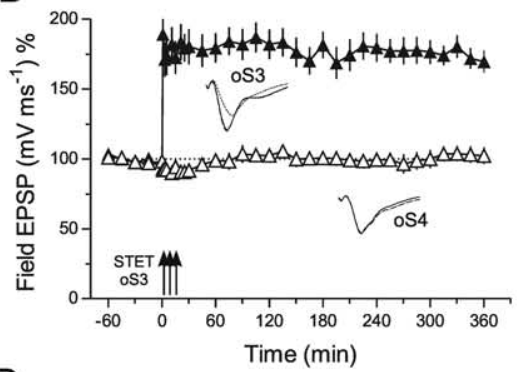

D

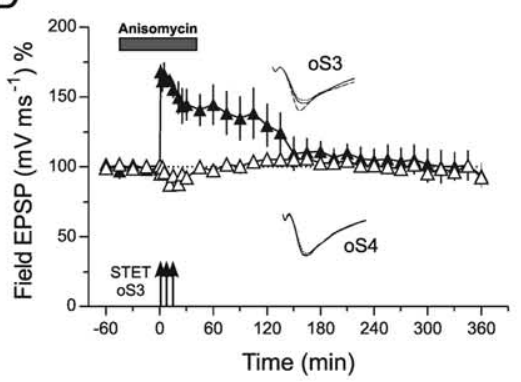

$\mathrm{F}$

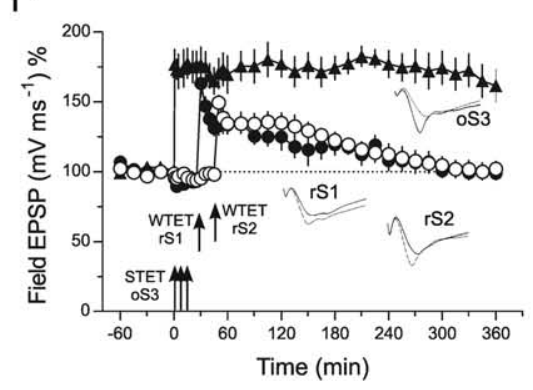

$\mathrm{H}$

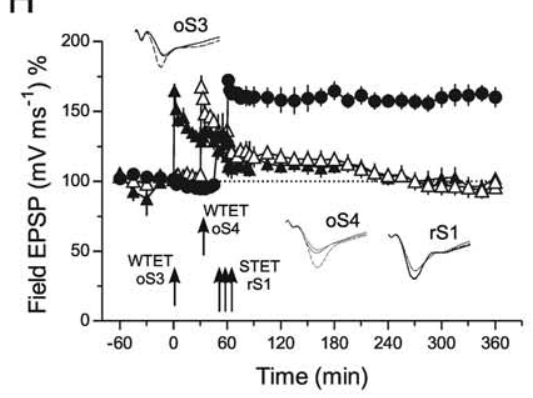

Figure 4. Restriction of synaptic tagging to neuronal functional compartments. The schematic figure in $\boldsymbol{A}$ represents the location of additional electrodes to stimulate independent basal synaptic input ( 053 and 054 ) to the CA1 pyramidal neuron and the positioning of a third field EPSP recording electrode in the area of the basal dendrites between stimulating electrodes $0 S 3$ and $0 S 4$. When the stratum oriens field EPSP was directly recorded, the electrode for recording the PS (dotted electrode) was omitted. The rest is as in Figure 1A. B. The time course of late LTP induced by a STET in OS3 (filled triangles) and the time course of control recordings in $0 S 4$ (open triangles; $n=8$ ). The transient maintenance of early LTP produced by a WTET to input $0 S 3$ is presented in
C (filled triangles; $n=9)$. Open triangles represent the time course of the control pathway oS4. D, E, Results with structurally different protein synthesis inhibitors ( $\boldsymbol{D}$, anisomycin, $n=$ 7; $\boldsymbol{E}$, emetine, $n=4$ ) when bath applied $45 \mathrm{~min}$ before STET (filled triangles) until 45 min after TET to basal 0S3. Early LTP (C) was slightly affected by anisomycin (D) but not by emetine (E). Our own unpublished work revealed a moderate unspecific effect of anisomycin but not emetine on PS recordings that could be related to the soma near unspecific effects of anisomycin, especially in the case of stratum oriens LTP. Therefore, the second structural inhibitor is required to support its specificity on protein synthesis. Open triangles represent the time course of the control pathway oS4.F, Induction of late LTP in OS3 (filled triangles), followed by WTET to the apical rS1 (30 min after STET to 0S3; filled circles) and apical rS2 (40 min after STET to 0S3; open circles). $n=7$. In $\mathbf{G}$, a similar set of experiments as in $\boldsymbol{F}$ is shown, with the difference that STET was applied to apical rS1 (filled circles) followed 30 min later by WTET to apical rS2 (open circles) and 40 min later by WTET to basal rS3 (filled triangles; $n=7$ ). $\boldsymbol{H}$, If early LTP was induced in the basal inputs 053 (filled triangles) and 054 (open triangles) before induction of late LTP in the stratum radiatum (rS1; filled circles), early LTP in the stratum oriens could not benefit from late LTP in the stratum radiatum, resulting in decremental LTP in the basal inputs $(n=7) . I$, Late LTD was induced in the apical rS1 by SLFS (filled circles), followed by WTET to the apical rS2 30 min later (open circles) and WTET to the basal $0 S 3$ (filled triangles) 40 min after SLFS to S1. $n=7$. Error bars indicate SEM. Symbols and analog

traces are as in Figure 1.

specificity effect in apical dendrites (i.e., U0126 to inhibit MAPKs). Surprisingly, and in contrast to LTP in the apical denfected by these inhibitors (Fig. $5 B, C$ ). However, as it is the case for radiatum LTP, the maintenance of oriens LTP was
prevented by the inhibition of PKA (Fig. 5D) [LTP returned to baseline $300 \mathrm{~min}$ and Wilcoxon test, respectively; $p<0.05$ ] and PKM $\zeta$ (Fig. 5E) (LTP also returned to baseline $300 \mathrm{~min}$ after TET of oS3; Mann-
Whitney $U$ test or Wilcoxon test, respectively; $p<0.05$ ) activity during LTP induction. The next question was how these
enzymes could be involved in processes of synaptic tagging.

\section{LTP in the stratum oriens: synaptic
tagging and LTP-specific tag molecules}

We have now investigated whether synap-
tic tagging occurs within basal dendritic compartments during LTP. Figure $6 \mathrm{~A}$ normally results in early LTP was induced in the basal input oS3 (open triangles) foltriangles) $45 \mathrm{~min}$ later. The normally early by subsequent induction of late LTP in oS4, supporting the fact that synaptic tag- 
A

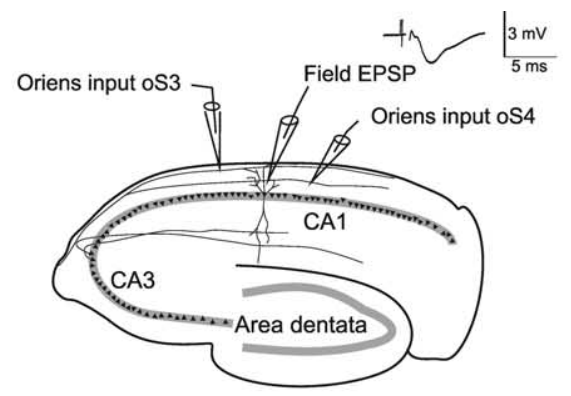

B

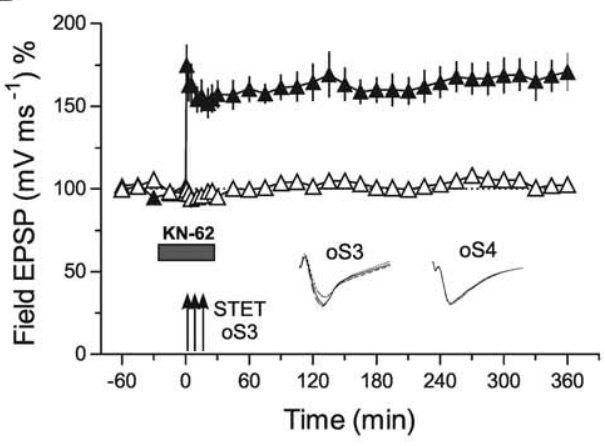

C

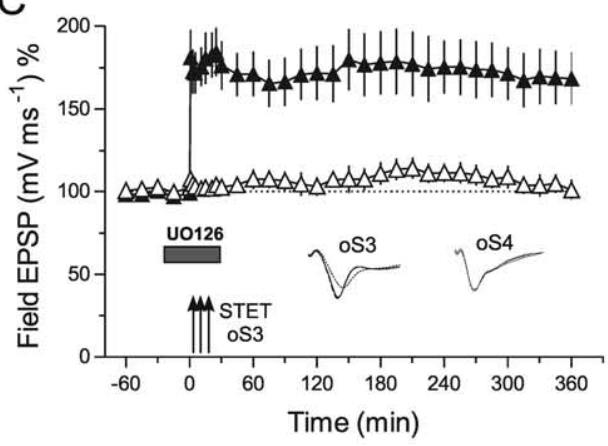

D
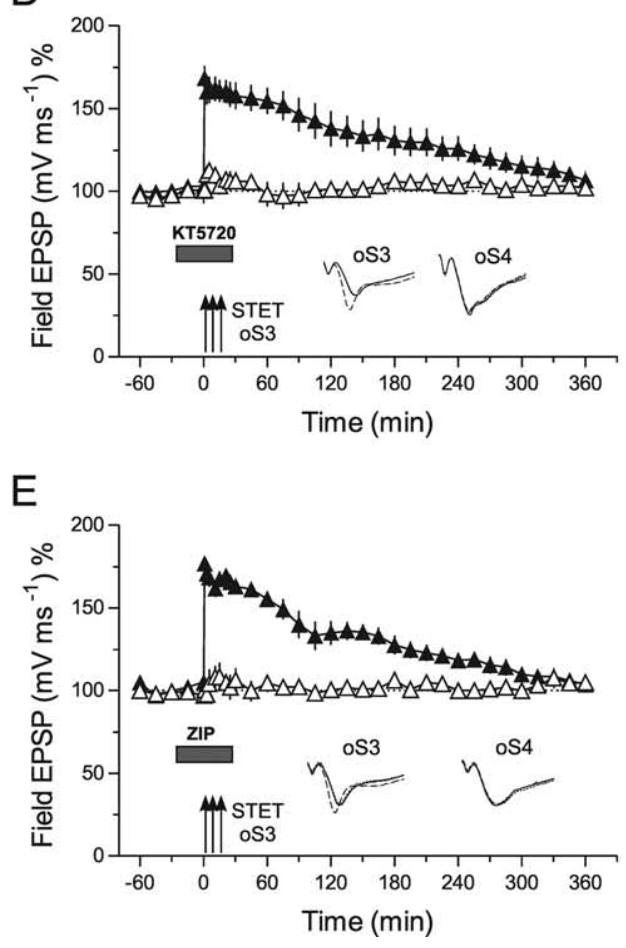

ging also takes place in the basal dendritic compartments of rat hippocampal pyramidal CA1 neurons. Next, we investigated whether inhibition of CaMKII (Fig. 6B) or MAPKs (Fig. 6C) specifically interferes with synaptic tagging in oS3. The inhibitors were applied during the WTET of oS3 and then rapidly washed out to assure that STET applied to oS4 was not influenced anymore by the drugs. If synaptic tags were set in oS3, these tags could capture PRPs produced by the late LTP in oS4, transforming the normally early LTP into late LTP in oS3. As shown in Figure $6 B, C$, CaMKII or MAPKs are not involved in setting synaptic tags in the basal dendritic region of hippocampal CA1 neurons, which is in contrast to the apical dendritic compartments represented in Figure 2. In search for other possible involved enzymes, we studied the role of PKA (Fig. 6D) and PKM $\zeta$ (Fig. $6 E$ ) for setting basal synaptic tags. Although KT5720 (PKA inhibitor) slightly affected the induction of early LTP in oS3 (Fig. 6D, open triangle), its transformation into late LTP was not prevented when late LTP was subsequently induced in a separate basal input oS4 (filled triangle). A similar result was obtained when ZIP, a specific PKM $\zeta$ inhibitor, was applied during the WTET of oS3 (Fig. 6E). Interestingly, if a mixture of the inhibitors for PKA and PKM $\zeta$ were applied during WTET of oS3, synaptic tagging in this input was prevented, resulting in no transformation of early LTP into late LTP of oS3 by subsequent induction of late LTP in oS4 (Fig. $6 F$ ). The potentiation in oS3 returned to baseline levels $240 \mathrm{~min}$ after WTET to oS3 (Wilcoxon test; $p<0.05)$. It remains unclear whether different mixtures (i.e., simultaneous inhibition of other enzymes) would also prevent the tag setting in basal dendrites. Additional studies need to be conducted to answer this question. It also remains unclear whether analogous processes in different animal species exist. It was shown, for instance, that PKA activity has a different function in mouse LTP induced in apical dendrites (Young et al., 2006).

To verify the specificity in preventing the setting of basal synaptic tags, we also investigated the potential role of PKA during synaptic tagging in the apical dendritic compartment (Fig. 6G-I). Similar to PKM $\zeta$ (Sajikumar et al., 2005b), PKA is not involved in processes of setting the tag in that compartment (i.e., the apical dendrites but affects the synthesis of PRPs) (Fig. 6G).

\section{Discussion}

CaMKs and MAPKs are multifunctional enzymes important for LTP and LTD only in apical dendrites and not in basal dendrites of hippocampal CA1 neurons. Both enzymes are required for the induction of late LTP/LTD. In addition to these general functions, however, ERK1/2 marks synaptic tags in an LTD specific way to capture PRPs and thus to transform early into late LTD. MAPKs, although involved in the maintenance of LTD and LTP, are not required for LTP tagging in apical dendrites. Similar results for MAPK were described for different forms of memory in Aplysia (Sharma et al., 2003). In contrast to the role of MAPKs, CaMKII is specific for processes occurring at LTP tags (i.e., it is

Figure 5. Late LTP and kinases in basal CA1-dendrites. $\boldsymbol{A}$, The schematic location of the electrodes in the stratum oriens (symbols as in Fig. 4). $\boldsymbol{B}$, Late LTP in basal dendrites is unaffected by inhibition of CaMKII by KN-62 when applied during its induction in oS3 (filled triangles). Control stimulation of oS4 revealed no effect of the drug on baseline potentials (open triangles; $n=6$ ). $C$, Similar to $B$, inhibition of MAPKs by U0126 was also ineffective in preventing late LTP in basal synaptic inputs $(n=7)$. However, application of the PKA inhibitor KT5720 (D) or the PKM $\zeta$ blocker ZIP (E) prevented the maintenance of LTP in 0 S3 (filled triangles) without affecting the control input oS4 (D,n=7; $\boldsymbol{E}, n=7)$. Error bars indicate SEM. Symbols and analog traces are as in Figure 1. 

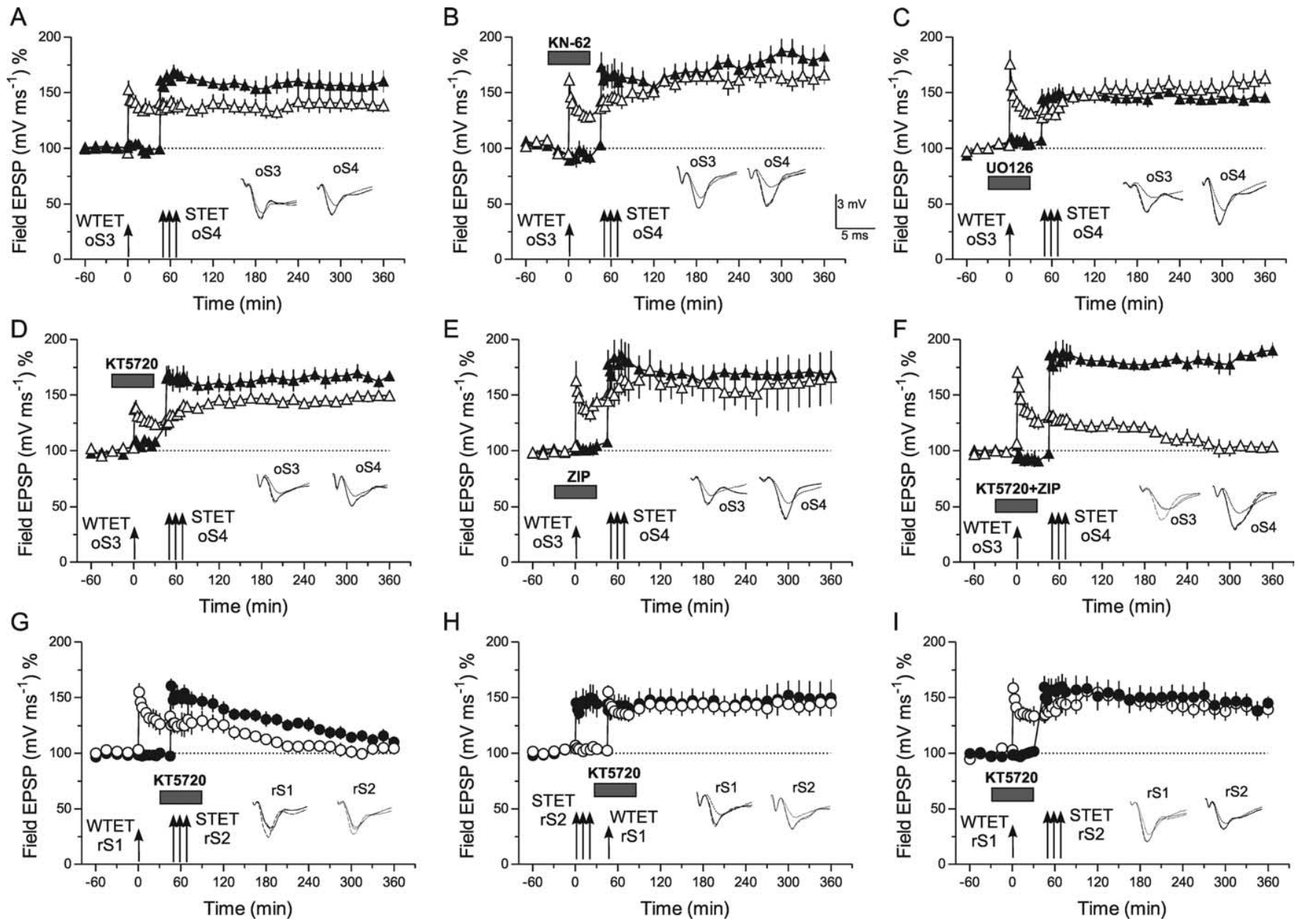

Figure 6. LTP in stratum oriens: synaptic tagging and LTP tag molecules. A, Early LTP was induced in the basal input oS3 by a WTET (open triangles) followed by a STET to basal input 0S4 (filled triangles) 45 min later. The normally early LTP in oS3 was transformed into late LTP by subsequent induction of late LTP in oS4 $(n=9)$. Inhibition of CaMKII by KN-62 (B, $n=8)$ or MAPKs by U0126 $(C, n=8)$ did not interfere with synaptic tagging in oS3 when the drug was applied 30 min before the induction of early LTP in oS3 (open triangles) and subsequently washed out ( 30 min after WTET) sufficiently before STET to OS4 (filled triangles). In $\boldsymbol{D}(n=7)$, the PKA inhibitor KT5720 was applied during the WTET of oS3 similarly as in $\boldsymbol{B}$ and $\boldsymbol{C}$; synaptic tagging in oS3 was not prevented by the drug and subsequent induction of late LTP to oS4 after washing out of KT5720 (filled triangles). $\boldsymbol{E}$, Experiment similar to that in $\boldsymbol{D}$, with the exception that instead of the PKA inhibitor, the PKM $\zeta$ blocker ZIP was applied. Also, here, synaptic tagging was not prevented $(n=8)$. If a mixture of the inhibitors for PKA and PKM $\zeta$ were applied during WTET of oS 3 shown in $F$, synaptic tagging in this input was prevented, resulting in no transformation of early LTP into late LTP of oS3 (open triangles) by subsequent induction of late LTP in oS4 (filled triangles; $n=8$ ). To verify the specificity in preventing the setting of basal synaptic tags, G-I represent experiments in which we investigated the potential role of PKA during synaptic tagging in the apical dendritic compartment. G, WTET in rS1 of apical dendrites was applied (open circles). Thirty minutes later, the PKA inhibitor KT5720 was applied, and another 30 min later, under the influence of the drug, STET was applied to rS2 (filled circles; $n=7$ ). Synaptic tagging did not take place; late LTP in $r S 2$ was prevented. In $\boldsymbol{H}$, the order of LTP induction was reversed: STET was induced in rS2 (filled circles) followed by the application of KT572030 min later. Another 30 min later (but now under PKA inhibition), WTET was applied to rS1 (open circles). Normally, this TET paradigm should only result in early LTP in rS2, but rS2 PRPs could be captured by the rS1 tags, thus transforming its early into late LTP $(n=7)$. I, Series of experiments similar to that in $G$, but now the PKA inhibitor was applied during WTET of rS1 (open circles) followed by induction of late LTP in rS2 (filled circles) without inhibition of PKA $(n=7)$. As it was the case in $\boldsymbol{H}$, if the synthesis of rS2 PRPs was not prevented, the rS2 tags (immune against PKA inhibition) could capture these PRPs and thus paradoxically transform its normally early LTP into late LTP. Error bars indicate SEM. Symbols and analog traces are as in Figure 1.

required to capture/process PRPs to ensure the transformation of early into late LTP in the apical dendrites).

MAPKs and CaMKs may exert a general role for the synthesis of PRPs throughout the prolonged maintenance of the plasticity events, most likely at restricted local dendritic sites (apical dendrites) (Sweatt, 2001; Miller et al., 2002; Kelleher et al., 2004a,b). This is supported by a continuous long-lasting activation of MAPKs during different phases of LTP (Ahmed and Frey, 2005), which is quite similar to the prolonged activation of CaMKII seen in some (Giovannini et al., 2001; Ahmed and Frey, 2005; Atkins et al., 2005) but not other (Chen et al., 2001; Lengyel et al., 2004) laboratories. The differences with respect to the prolonged phosphorylation of CaMKII could be explained by methodological issues (Sajikumar et al., 2005a; Cooke et al., 2006). The MAPK and CaMK family may exert various effects during plasticity events induced in the apical dendrites. Our findings obtained in basal dendrites are in contrast to the role of MAPKs and CaMKII in apical dendrites. In the stratum oriens, MAPK or CaMK inhibitors are ineffective in interfering with LTP (Fig. 5) or synaptic tagging (Fig. 6). Strikingly, PKA as well as PKM $\zeta$, which have a specific function for LTP maintenance in the apical dendrites (Frey et al., 1993; Sajikumar et al., 2005b), are required for the induction of late LTP and synaptic tagging in the basal dendrites. In apical dendrites, PKA activity is specifically required for the induction of the synthesis of PRPs (Frey et al., 1993) (Fig. 6G-I), whereas PKM $\zeta$ represents an LTP-specific PRP (Sajikumar et al., 2005b). In addition to the function of both enzymes in the apical dendrites, they are involved in activating the tagging process in basal dendrites. It remains to be investigated by which pathway each of these enzymes is activated, and whether both enzymes are 
also involved in the induction of the synthesis of PRPs in the basal dendrites. The region-specific differences in the cellular functional pathways used for the induction of lasting plasticity events could be explained by the various information processed in these compartments via different activity patterns resulting in the activation of different receptors/channels including voltage-dependent calcium channels and/or neuromodulatory inputs.

Our data show that even the computationally interesting and novel property of late-associative interactions during synaptic tagging, cross-tagging, as well as compartment-specific plasticity follows specific rules for both establishing and maintaining its functional specificity. Thus, in addition to process- and compartment-specific PRPs, such as PKM $\zeta$ for LTP in apical dendrites, there are process- and compartment-specific tags to guarantee the expression of one long-term plasticity event at a particular synapse. Thus, a specific interaction between CaMKII and PKM $\zeta$ in apical dendrites could be responsible for the synapse-specific expression of late LTP there. However, the systematic integration of afferent information at the cellular level during encoding and consolidation of memory is determined by the restriction of the tagging processes to local dendritic compartments and by compartmentspecific different intracellular pathways.

Our results support the hypothesis that instead of formation of a memory trace within a single synapse, functional synaptic populations (i.e., functional integrative compartments) exist within neural networks (Mel, 1993; Bonhoeffer, 1997; Kelleher et al., 2004b; Polsky et al., 2004; Alarcon et al., 2006; Engert and Govindarajan et al., 2006; Reymann and Frey, 2007). We suggest that tagging processes are locally restricted for a more efficient and reliable controlling of systemic and organism-relevant information processing (Frey, 2001; Polsky et al., 2004; Sajikumar et al., 2005b). However, recent work from others suggests that this local restriction to functional compartments can be overcome under distinct circumstances.

What is the function of dendritic compartmentalization versus uncompartmentalization of synaptic plasticity to the mammal's behavior? We favor a model that is supported by our work in intact behaving animals (Frey et al., 2001; Korz and Frey, 2004): under normal, nonlifethreatening situations, a restricted, compartmentalized regulation of PRPs is achieved by specific neuromodulatory in-

\section{Compartmentalized Synaptic Tagging and Cross-Tagging}
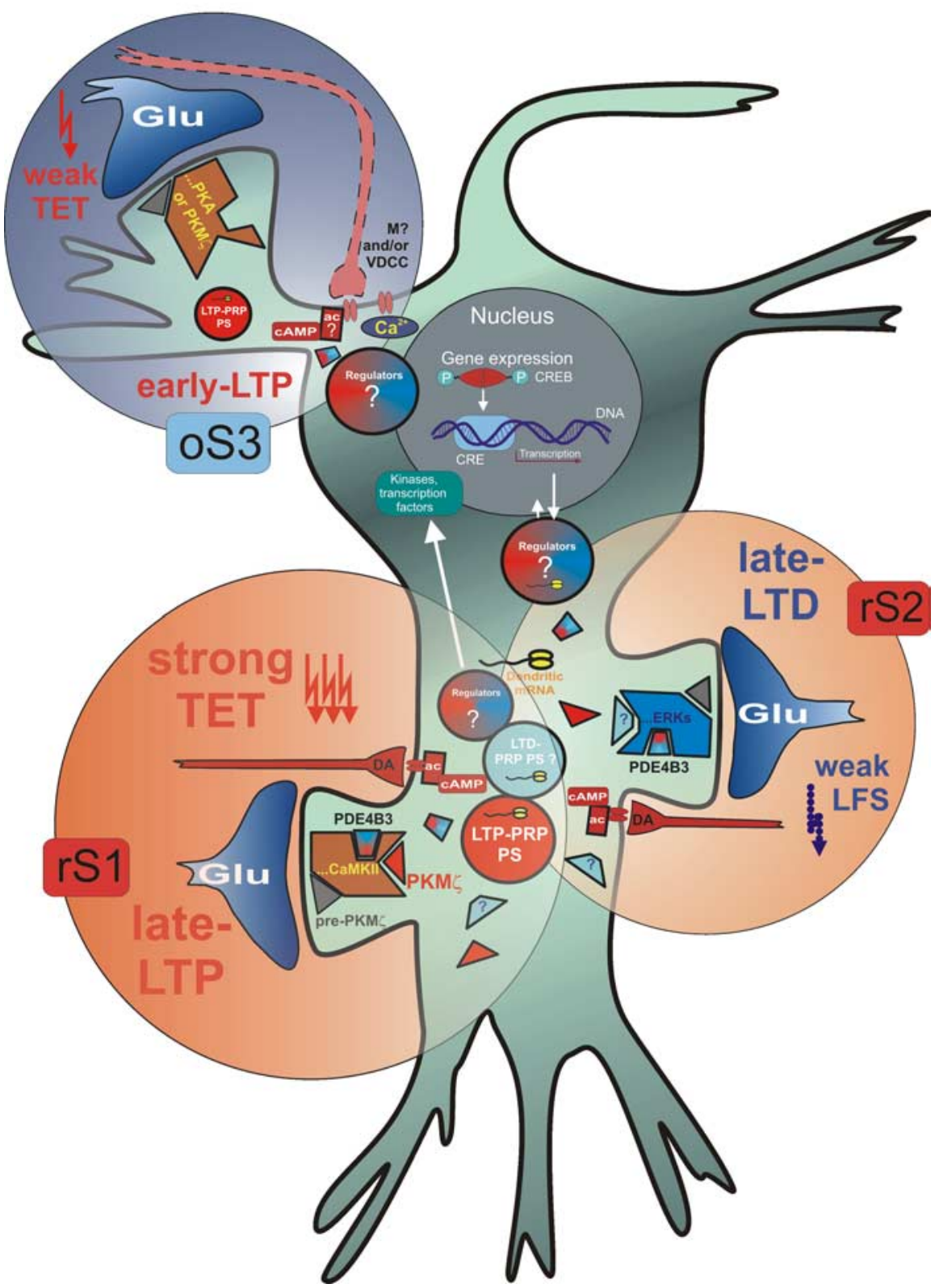

Figure 7. Scheme representing the theory of compartmentalized synaptic tagging and cross-tagging in a hippocampal pyramidal CA1 neuron. The induction of late LTP (and similarly late LTD) in the apical synaptic input rS1 activates postsynaptically via a heterosynaptic synergistic interaction of glutamatergic and dopaminergic receptors. A transient, protein synthesis-independent synaptic tag, which is made specific for LTP by CaMKII-dependent processes and the synthesis of a pool of PRPs. PRPs consist of LTP-specific proteins, in particular PKM $\zeta$, regulatory proteins such as phosphodiesterase type 4B3 (PDE4B3), and most likely not yet identified LTD-specific PRPs. Both the tag as well as the PRPs have a distinct half-life. During that time window, the interaction between the two must take place, maintaining LTP in $\mathrm{rS} 1$ for $\geq 8 \mathrm{~h}$. If, within the time window of available PRPs and within the dendritic compartment, a second independent synaptic input is stimulated to set a synaptic tag (e.g., by inducing early LTD or early LTP), the latter can benefit from the compartment-restricted availability of PRPs, thus transforming the normally transient plasticity form into an enduring one. In the example shown, early LTD is transformed into late LTD by processes of cross-tagging or capturing of the PRPs provided through input rS1. If, within the same time window at a basal dendritic compartment 0 S3, an early event is induced even with a synaptic tag set (here, early LTP by a WTET), these tags cannot benefit because the availability of PRPs is compartmentalized in the apical dendrites, thus resulting in a transient expression of early LTP at basal inputs. Crosscompartmental tagging does not occur. Interestingly, setting of synaptic tags and LTP expression in synapses of the basal dendrites require the action of different enzymes as in the apical dendrites. Where CaMKII is a molecule that mediates LTP-specific tags in the stratum radiatum, the setting of LTP-specific tags in stratum oriens requires the PKA or PKM $\zeta$ activity but not necessarily the two together at the same time. PKM $\zeta$ is an LTP-specific PRP in the apical dendrites; however, whether it has a dual function in basal dendrites remains to be investigated. Similarly, it remains to be investigated which molecular key players are relevant for stratum oriens LTD. It also remains unclear if basal plasticity events require nonglutamatergic modulatory inputs. For a prolonged main- 
puts (e.g., dopaminergic inputs at apical dendrites) (Fig. 7). In contrast, the processing of very important information for the organism, like a life-threatening event with a high emotional or cognitive content, requires information storage in a large number of neuronal networks by losing compartment specificity. Basal dendrites with their close relation to the soma (Fig. 7) may regulate a neuron-wide synthesis of PRPs, possibly via a direct activation of gene expression (Dudek and Fields, 2002; Adams and Dudek, 2005; Reymann and Frey, 2007), thus providing a cellular mechanism for a general increase in the capacity to store relevant information within the neuron.

Our results allow us to further develop the hypothesis of compartmentalized memory consolidation at the cellular level (Frey, 2001; Korz and Frey, 2004; Sajikumar and Frey, 2004a; Sajikumar et al., 2005b; Reymann and Frey, 2007). We suggest that processing, encoding, and consolidation of a memory trace under nonlife-threatening situations takes place in functional dendritic compartments (Frey and Morris, 1998a; Sajikumar and Frey, 2004a). The functional relevance of compartmentalized cellular memory formation is determined by the physiological relevance of the afferent information. As described previously (Morris and Frey, 1997; Frey and Morris, 1998a; Frey, 2001; Korz and Frey, 2004; Sajikumar and Frey, 2004a; Sajikumar et al., 2005b), in the intact animal, an afferent stimulus might be automatically and transiently stored at glutamatergic synapses (e.g., by processes of early LTP/LTD), regardless of their final relevance for the organism. However, if the system decides that the information is relevant to be stored more permanently, within the time window of $\sim 30 \mathrm{~min}$, the system can regulate PRP synthesis in an associative manner within distinct functional compartments to transfer the transient memory into a long-lasting one. This can happen by modulatory dopaminergic signals or other neuromodulators to compartments or neuron wide by stress hormones. Figure 7 summarizes our concept of cellular memory formation in a pyramidal CA1 neuron.

The here described new properties of late-plasticity events in hippocampal neurons support a new principle of cellular information storage (i.e., the idea of a "compartmentalized processing and information storage") (Frey, 2001; Reymann and Frey, 2007). This general idea was already outlined in more theoretical papers (Mel, 1993; Polsky et al., 2004) and was further developed by the "clustered engram" theory (Govindarajan et al., 2006), in which it was proposed that these cellular mechanisms of information processing represent a general principle of long-term information storage. Our data support these hypotheses and provide the first experimental evidence for the existence of functional clusters during cellular information storage by studying processes of hippocampal plasticity. Recent results using functional magnetic resonance imaging in humans suggest similar events in humans and strengthen the above assumptions (Schott et al., 2004; Wittmann et al., 2005). Many questions remain, for instance, the impact of LTD induced in the basal inputs to other compartments. Another question is whether the apical dendrites can be further divided into more functional compartments by a rather localized stimulation of dendritic branches, etc.

\section{References}

Adams JP, Dudek SM (2005) On the late-phase of LTP: getting to the nucleus. Nat Rev Neurosci 6:737-743.

Ahmed T, Frey JU (2003) Expression of the specific type IV phosphodiesterase gene PDE4B3 during different phases of long-term potentiation in single hippocampal slices of rats in vitro. Neuroscience 117:627-638.

Ahmed T, Frey JU (2005) Plasticity-specific phosphorylation of CaMKII, MAP-kinases and CREB during late-LTP in rat hippocampal slices in vitro. Neuropharmacology 49:477-492.

Ahmed T, Frey S, Frey JU (2004) Regulation of the phosphodiesterase PDE4B3-isotype during long-term potentiation in the area dentata in vivo. Neuroscience 124:857-867.

Alarcon JM, Barco A, Kandel ER (2006) Capture of the late phase of long-term potentiation within and across the apical and basilar dendritic compartments of CA1 pyramidal neurons: synaptic tagging is compartment restricted. J Neurosci 26:256-264.

Alkon DL, Amaral DG, Bear MF, Black J, Carew TJ, Cohen NJ, Disterhoft JF, Eichenbaum H, Golski S, Gorman LK, Lynch G, McNaughton BL, Mishkin M, Moyer JRJ, Olds JL, Olton DS, Otto T, Squire LR, Staubli U, Thompson LT, et al. (1991) Learning and memory. Brain Res Rev 16:193-220.

Amaral DG, Kurz J (1985) An analysis of the origins of the cholinergic and noncholinergic septal projections to the hippocampal formation of the rat. J Comp Neurol 240:37-59.

Amaral DG, Witter MP (1998) Hippocampal formation. In: The rat nervous system (Paxinos G, ed), pp 443-493. San Diego: Academic.

Atkins CM, Davare MA, Oh MC, Derkach V, Soderling TR (2005) Bidirectional regulation of cytoplasmic polyadenylation elementbinding protein phosphorylation by $\mathrm{Ca}^{2+} /$ calmodulin-dependent protein kinase II and protein phosphatase 1 during hippocampal long-term potentiation. J Neurosci 25: 5604-5610. 
Barco A, Alarcon JM, Kandel ER (2002) Expression of constitutively active CREB protein facilitates the late phase of long-term potentiation by enhancing synaptic capture. Cell 108:689-703.

Bortolotto ZA, Collingridge GL (1998) Involvement of calcium/ calmodulin-dependent protein kinases in the setting of a molecular switch involved in hippocampal LTP. Neuropharmacology 37:535-544.

Casadio A, Martin KC, Giustetto M, Zhu H, Chen M, Bartsch D, Bailey CH, Kandel ER (1999) A transient, neuron-wide form of CREB-mediated long-term facilitation can be stabilized at specific synapses by local protein synthesis. Cell 99:221-237.

Chen HX, Otmakhov N, Strack S, Colbran RJ, Lisman JE (2001) Is persistent activity of calcium/calmodulin-dependent kinase required for the maintenance of LTP? J Neurophysiol 85:1368-1376.

Cooke SF, Wu J, Plattner F, Errington M, Rowan M, Peters M, Hirano A, Bradshaw KD, Anwyl R, Bliss T, Giese KP (2006) Autophosphorylation of alphaCaMKII is not a general requirement for NMDA receptordependent LTP in the adult mouse. J Physiol (Lond) 574:805-818.

Dudek SM, Fields RD (2001) Mitogen-activated protein kinase/extracellular signal-regulated kinase activation in somatodendritic compartments: roles of action potentials, frequency, and mode of calcium entry. J Neurosci 21:RC122(1-5).

Dudek SM, Fields RD (2002) Somatic action potentials are sufficient for late-phase LTP-related cell signaling. Proc Natl Acad Sci USA 99:3962-3967.

Engert F, Bonhoeffer T (1997) Synapse specificity of long-term potentiation breaks down at short distances. Nature 388:279-284.

English JD, Sweatt JD (1997) A requirement for the mitogen-activated protein kinase cascade in hippocampal long term potentiation. J Biol Chem 272:19103-19106.

Fonseca R, Nägerl UV, Morris RG, Bonhoeffer T (2004) Competing for memory: hippocampal LTP under regimes of reduced protein synthesis. Neuron 44:1011-1020.

Frey JU (2001) Long-lasting hippocampal plasticity: cellular model for memory consolidation? In: Cell polarity and subcellular RNA localization (Richter D, ed), pp. 27-40. Berlin: Springer.

Frey S, Bergado-Rosado J, Seidenbecher T, Pape HC, Frey JU (2001) Reinforcement of early long-term potentiation (early-LTP) in dentate gyrus by stimulation of the basolateral amygdala: heterosynaptic induction mechanisms of late-LTP. J Neurosci 21:3697-3703.

Frey U (1997) Cellular mechanisms of long-term potentiation: late maintenance. In: Neural network models of cognition: biobehavioral foundations (Donahoe JW, Dorsel VP, eds), pp 105-128. Amsterdam: Elsevier Science.

Frey U, Morris RG (1997) Synaptic tagging and long-term potentiation. Nature 385:533-536.

Frey U, Morris RG (1998a) Synaptic tagging: implications for late maintenance of hippocampal long-term potentiation. Trends Neurosci 21:181-188.

Frey U, Morris RG (1998b) Weak before strong: dissociating synaptic tagging and plasticity- factor accounts of late-LTP. Neuropharmacology 37:545-552.

Frey U, Krug M, Reymann KG, Matthies H (1988) Anisomycin, an inhibitor of protein synthesis, blocks late phases of LTP phenomena in the hippocampal CA1 region in vitro. Brain Res 452:57-65.

Frey U, Huang YY, Kandel ER (1993) Effects of cAMP simulate a late stage of LTP in hippocampal CA1 neurons. Science 260:1661-1664.

Giovannini MG, Blitzer RD, Wong T, Asoma K, Tsokas P, Morrison JH, Iyengar R, Landau EM (2001) Mitogen-activated protein kinase regulates early phosphorylation and delayed expression of $\mathrm{Ca}^{2+} /$ calmodulindependent protein kinase II in long-term potentiation. J Neurosci 21:7053-7062.

Govindarajan A, Kelleher RJ, Tonegawa S (2006) A clustered plasticity model of long-term memory engrams. Nat Rev Neurosci 7:575-583.

Kauderer BS, Kandel ER (2000) Capture of a protein synthesis-dependent component of long-term depression. Proc Natl Acad Sci USA 97:13342-13347.

Kelleher III RJ, Govindarajan A, Jung HY, Kang HJ, Tonegawa S (2004a) Translational control by MAPK signaling in long-term synaptic plasticity and memory. Cell 116:467-479.

Kelleher III RJ, Govindarajan A, Tonegawa S (2004b) Translational regulatory mechanisms in persistent forms of synaptic plasticity. Neuron 44:59-73.
Komiyama NH, Watabe AM, Carlisle HJ, Porter K, Charlesworth P, Monti J, Strathdee DJ, O'Carroll CM, Martin SJ, Morris RG, O’Dell TJ, Grant SG (2002) SynGAP regulates ERK/MAPK signaling, synaptic plasticity, and learning in the complex with postsynaptic density 95 and NMDA receptor. J Neurosci 22:9721-9732.

Korz V, Frey JU (2004) Emotional and cognitive reinforcement of rat hippocampal long-term potentiation by different learning paradigms. Neuron Glia Biol 1:253-261.

Krug M, Lössner B, Ott T (1984) Anisomycin blocks the late phase of longterm potentiation in the dentate gyrus of freely moving rats. Brain Res Bull 13:39-42.

Lee HK, Barbarosie M, Kameyama K, Bear MF, Huganir RL (2000) Regulation of distinct AMPA receptor phosphorylation sites during bidirectional synaptic plasticity. Nature 405:955-959.

Lengyel I, Voss K, Cammarota M, Bradshaw K, Brent V, Murphy KP, Giese KP, Rostas JA, Bliss TV (2004) Autonomous activity of CaMKII is only transiently increased following the induction of long-term potentiation in the rat hippocampus. Eur J Neurosci 20:3063-3072.

Lisman JE, Grace AA (2005) The hippocampal-VTA loop: controlling the entry of information into long-term memory. Neuron 46:703-713.

Malinow R, Madison DV, Tsien RW (1988) Persistent protein kinase activity underlying long-term potentiation. Nature 335:820-824.

Malinow R, Schulman H, Tsien RW (1989) Inhibition of postsynaptic PKC or CaMKII blocks induction but not expression of LTP. Science 245:862-866.

Manahan-Vaughan D, Kulla A, Frey JU (2000) Requirement of translation but not transcription for the maintenance of long-term depression in the CA1 region of freely moving rats. J Neurosci 20:8572-8576.

Martin KC, Barad M, Kandel ER (2000) Local protein synthesis and its role in synapse-specific plasticity. Curr Opin Neurobiol 10:587-592.

Matthies H (1989) In search of cellular mechanisms of memory. Prog Neurobiol 32:277-349.

Mayford M, Wang J, Kandel ER, O’Dell TJ (1995) CaMKII regulates the frequency-response function of hippocampal synapses for the production of both LTD and LTP. Cell 81:891-904.

Mel BW (1993) Synaptic integration in an excitable dendritic tree. J. Neurophysiol 70:1086-1101.

Miller S, Yasuda M, Coats JK, Jones Y, Martone ME, Mayford M (2002) Disruption of dendritic translation of CaMKII $\alpha$ impairs stabilization of synaptic plasticity and memory consolidation. Neuron 36:507-519.

Morris RG, Frey U (1997) Hippocampal synaptic plasticity: role in spatial learning or the automatic recording of attended experience? Philos Trans R Soc Lond B Biol Sci 352:1489-1503.

Navakkode S, Sajikumar S, Frey JU (2004) The type IV-specific phosphodiesterase inhibitor rolipram and its effect on hippocampal long-term potentiation and synaptic tagging. J Neurosci 24:7740-7744.

Navakkode S, Sajikumar S, Frey JU (2005) Mitogen-activated protein kinase-mediated reinforcement of hippocampal early long-term depression by the type IV-specific phosphodiesterase inhibitor rolipram and its effect on synaptic tagging. J Neurosci 25:10664-10670.

Otani S, Marshall CJ, Tate WP, Goddard GV, Abraham WC (1989) Maintenance of long-term potentiation in rat dentate gyrus requires protein synthesis but not messenger RNA synthesis immediately posttetanization. Neuroscience 28:519-526.

Pikkarainen M, Rönkkö S, Savander V, Insausti R, Pitkänen A (1999) Projections from the lateral, basal, and accessory basal nuclei of the amygdala to the hippocampal formation in rat. J Comp Neurol 403:229-260.

Polsky A, Mel BW, Schiller J (2004) Computational subunits in thin dendrites of pyramidal cells. Nat Neurosci 7:621-627.

Reymann KG, Frey JU (2007) The late maintenance of hippocampal LTP: requirements, phases, 'synaptic tagging,' 'late-associativity' and implications. Neuropharmacology 52:24-40.

Sajikumar S, Frey JU (2003) Anisomycin inhibits the late maintenance of long-term depression in rat hippocampal slices in vitro. Neurosci Lett 338:147-150.

Sajikumar S, Frey JU (2004a) Late-associativity, synaptic tagging, and the role of dopamine during LTP and LTD. Neurobiol Learn Mem 82:12-25.

Sajikumar S, Frey JU (2004b) Resetting of 'synaptic tags' is time- and activity-dependent in rat hippocampal Cal in vitro. Neuroscience 129:503-507.

Sajikumar S, Navakkode S, Frey JU (2005a) Protein synthesis-dependent 
long-term functional plasticity: methods and techniques. Curr Opin Neurobiol 15:607-613.

Sajikumar S, Navakkode S, Sacktor TC, Frey JU (2005b) Synaptic tagging and cross-tagging: the role of protein kinase $\mathrm{M} \zeta$ in maintaining long-term potentiation but not long-term depression. J Neurosci 25:5750-5756.

Schnabel R, Palmer MJ, Kilpatrick IC, Collingridge GL (1999) A CaMKII inhibitor, KN-62, facilitates DHPG-induced LTD in the CA1 region of the hippocampus. Neuropharmacology 38:605-608.

Schott BH, Sellner DB, Lauer CJ, Habib R, Frey JU, Guderian S, Heinze HJ, Duzel E (2004) Activation of midbrain structures by associative novelty and the formation of explicit memory in humans. Learn Mem 11:383-387.

Sharma SK, Sherff CM, Shobe J, Bagnall MW, Sutton MA, Carew TJ (2003) Differential role of mitogen-activated protein kinase in three distinct phases of memory for sensitization in Aplysia. J Neurosci 23:3899-3907.

Stanton PK, Gage AT (1996) Distinct synaptic loci of $\mathrm{Ca}^{2+} /$ calmodulindependent protein kinase II necessary for long-term potentiation and depression. J Neurophysiol 76:2097-2101.

Stevens CF, Tonegawa S, Wang Y (1994) The role of calcium-calmodulin kinase II in three forms of synaptic plasticity. Curr Biol 4:687-693.

Steward O (2002a) Local synthesis of proteins at synaptic sites on dendrites: role in synaptic plasticity and memory consolidation? Neurobiol Learn Mem 78:508-527.

Steward O (2002b) mRNA at synapses, synaptic plasticity, and memory consolidation. Neuron 36:338-340.

Steward O, Halpain S (1999) Lamina-specific synaptic activation causes domain-specific alterations in dendritic immunostaining for MAP2 and CAM kinase II. J Neurosci 19:7834-7845.
Steward O, Schuman EM (2001) Protein synthesis at synaptic sites on dendrites. Annu Rev Neurosci 24:299-325.

Steward O, Wallace CS, Lyford GL, Worley PF (1998) Synaptic activation causes the mRNA for the IEG Arc to localize selectively near activated postsynaptic sites on dendrites. Neuron 21:741-751.

Sweatt JD (2001) The neuronal MAP kinase cascade: a biochemical signal integration system subserving synaptic plasticity and memory. J Neurochem 76:1-10.

Sweatt JD (2004) Mitogen-activated protein kinases in synaptic plasticity and memory. Curr Opin Neurobiol 14:311-317.

Tompa P, Friedrich P (1998) Synaptic metaplasticity and the local charge effect in postsynaptic densities. Trends Neurosci 21:97-102.

Winder DG, Mansuy IM, Osman M, Moallem TM, Kandel ER (1998) Genetic and pharmacological evidence for a novel, intermediate phase of long-term potentiation suppressed by calcineurin. Cell 92:25-37.

Winder DG, Martin KC, Muzzio IA, Rohrer D, Chruscinski A, Kobilka B, Kandel ER (1999) ERK plays a regulatory role in induction of LTP by theta frequency stimulation and its modulation by $\beta$-adrenergic receptors. Neuron 24:715-726.

Wittmann BC, Schott BH, Guderian S, Frey JU, Heinze HJ, Düzel E (2005) Reward-related fMRI activation of dopaminergic midbrain is associated with enhanced hippocampus-dependent long-term memory formation. Neuron 45:459-467.

Young JZ, Nguyen PV (2005) Homosynaptic and heterosynaptic inhibition of synaptic tagging and capture of long-term potentiation by previous synaptic activity. J Neurosci 25:7221-7231.

Young JZ, Isiegas C, Abel T, Nguyen PV (2006) Metaplasticity of the latephase of long-term potentiation: a critical role for protein kinase A in synaptic tagging. Eur J Neurosci 23:1784-1794. 